\title{
Pulmonary infections in patients with and without hematological malignancies: diagnostic yield and safety of flexible bronchoscopy - a retrospective analysis
}

\author{
Jens Panse ${ }^{1}$, Kai von Schwanewede ${ }^{2}$, Edgar Jost ${ }^{1}$, Michael Dreher $^{2}$, Tobias Müller ${ }^{2} \wedge$ \\ ${ }^{1}$ Department of Hematology, Oncology, Hemostaseology and Stem Cell Transplantation, ${ }^{2}$ Department of Pneumology and Intensive Care Medicine, \\ University Hospital RWTH Aachen, Aachen, Germany \\ Contributions: (I) Conception and design: J Panse, E Jost, T Müller; (II) Administrative support: None; (III) Provision of study materials or patients: \\ None; (IV) Collection and assembly of data: K von Schwanewede, T Müller; (V) Data analysis and interpretation: K von Schwanewede, M Dreher, T \\ Müller; (VI) Manuscript writing: All authors; (VII) Final approval of manuscript: All authors. \\ Correspondence to: Tobias Müller. Department of Pneumology and Intensive Care Medicine, University Hospital RWTH Aachen, Pauwelsstrasse 30, \\ 52074 Aachen, Germany. Email: tobmueller@ukaachen.de.
}

\begin{abstract}
Background: Fiberoptic bronchoscopy (FOB) with broncho-alveolar lavage (BAL) is frequently performed in patients with hematological malignancies and pulmonary opacities. While the safety of the procedure in this patient population has been shown, data about the diagnostic yield widely differ between studies. Furthermore, data comparing diagnostic yield and safety of flexible bronchoscopy to narrow sources of pulmonary infections in patients with and without underlying hematological malignancy are lacking.

Methods: We carried out a retrospective analysis of bronchoscopies done for the diagnostic work-up of pulmonary infections. Diagnostic yield and the occurrence of complications in patients with and without hematological disease were compared.

Results: In total $\mathrm{n}=268$ bronchoscopies were done in patients suffering from a hematological malignancy (HM) compared to $\mathrm{n}=408$ bronchoscopies in patients without hematological malignancy (NHM). The overall diagnostic yield was similar and did not differ between the groups (HM: 67.2\% vs. NHM: 64.7\%; $\mathrm{P}=0.5622$ ). However, when cultures positive for Candida were not considered as clinically relevant diagnostic yield was higher in the HM group (HM: 62.7\% vs. NHM: 53.9\%; P=0.0261) due to a higher detection rate of fungi and viruses (both $\mathrm{P}<0.001$ ). Interestingly, the diagnostic yield for bacteria was not decreased by pretreatment with antibiotics in either group (both $\mathrm{P}>0.05$ ). There was no difference in the complication rate between the groups and most complications were considered as minor.

Conclusions: In summary, our data demonstrate similar diagnostic yield and safety of flexible bronchoscopy for diagnosing pulmonary infection in patients with and without underlying hematological malignancy.
\end{abstract}

Keywords: Bronchoscopy; broncho-alveolar lavage (BAL); pulmonary infections; hematological malignancy

Submitted Feb 10, 2020. Accepted for publication Jul 04, 2020.

doi: $10.21037 /$ jtd-20-835

View this article at: http://dx.doi.org/10.21037/jtd-20-835

\footnotetext{
^ ORCID: 0000-0002-9801-3496.
} 


\section{Introduction}

Respiratory complications such as pulmonary infections frequently occur in patients suffering from hematological malignancies. Causes for respiratory complications, apart from opportunistic infections include chemo- or radiotherapy induced lung toxicity, invasion by malignant cells, graft-versus-host-disease, and increasingly immunological side effects such as pneumonitis due to the incremental availability of immune based therapies for this patient group. Risk factors for respiratory complications include underlying pulmonary diseases and active smoking $(1,2)$.

Fiberoptic bronchoscopy (FOB) with broncho-alveolar lavage (BAL) is commonly performed in hematological patients with pulmonary opacities and suspected pulmonary infection. In general, BAL is thought to provide valuable diagnostic information and an acceptable safety profile for FOB in this patient population has been demonstrated although a recent study found an association of FOB with increased hospital mortality in immunocompromised patients suffering from respiratory failure $(3,4)$. The diagnostic yield for the identification of infectious agents by $\mathrm{FOB}$ with BAL shows a considerable variation within different studies as broad-spectrum antibiotics are routinely administered before the initiation of FOB in patients with suspected pulmonary infections and hematologic malignancies (3,5-7). In addition, most studies investigating the diagnostic outcome of FOB with BAL included only a small number of patients and so far, no data are available comparing diagnostic yield and safety of FOB for suspected pulmonary infection in patients with and without hematological disease. Hence, the aim of this retrospective analysis was to analyze safety and diagnostic yield of FOB for the diagnosis of pulmonary infections in two large patient cohorts with (HM) and without underlying hematological malignancy (NHM).

\section{Methods}

Data analysis was done with regard to the Declaration of Helsinki (as revised in 2013). The Institutional Ethical Review Board for Human Studies at RWTH ("RheinischWestfälische Technische Hochschule") University was involved and confirmed that a formal approval was not required as this retrospective analysis required neither an intervention nor irregularity of privacy or anonymity (EK 099/17). Individual patient consent was waived by the Review Board due to the observational nature of the study.
An analysis of all flexible bronchoscopies for the evaluation of suspected pulmonary infection between January 2013 and December 2017 performed at the department of Pneumology at the University Hospital RWTH Aachen was conducted. The indication for bronchoscopy in aplastic patients with underlying hematological malignancies in general followed the recommendations of the German Infectious Disease Working Party (AGIHO) of the German Association for Hematology and Oncology (DGHO) (8-10). Briefly, CT scans are performed early in the disease process and if these reveal signs of infection/infiltration bronchoscopy/ BAL are performed if the patient's general condition allows so. In patients who are not aplastic the indication for bronchoscopy is made individually based on the clinical history, laboratory findings and imaging (typically CT scans).

Bronchoscopies of patients with critical illnesses (e.g., patients with acute respiratory failure or patients needing vasopressor support) were performed on intensive care or intermediate care wards and were not included in the analysis. All bronchoscopies were performed by boardcertified specialists in pulmonary or internal medicine experienced in bronchoscopy or under their direct supervision. All physicians performing bronchoscopy were trained and experienced in airway management (endotracheal intubation by direct laryngoscopy and by FOB), as well as in the management of acute emergency situations (e.g., shock, cardiac arrhythmias, or acute respiratory failure) and critical care medicine. In case of complications a second physician experienced in bronchoscopy, as well as an emergency team from the ICU were available on short notice. Standard monitoring included electrocardiogram, oxygen saturation $\left(\mathrm{SpO}_{2}\right)$, and non-invasive blood pressure (NIBP).

To increase patient tolerance and cooperation patients were sedated during the procedure typically by a combination of midazolam, fentanyl and propofol. In addition, lidocaine was administered topically to the vocal cords and the bronchial system. Bronchoalveolar lavage was performed using flexible bronchoscopes (Olympus, Japan). The bronchoscope was wedged into a segment where lung infiltration was present, or the infiltration was most severe. A total of $100 \mathrm{~mL}$ sterile saline solution was instilled in aliquots of $20 \mathrm{~mL}$ and the fluid was recovered by gentle aspiration. Original data was retrieved from an electronic patient record system (medico, Siemens, Germany) as well as from paper-based medical records and was collected in 
a Microsoft Access database (Microsoft, Redmond, USA), as described previously $(11,12)$. Demographic (age, sex) and epidemiological data (e.g., cardio-vascular or chronic pulmonary co-morbidities) were recorded, as well as data about underlying hematological malignancies, any form of immunosuppression, the amount of administered sedative drugs (midazolam, fentanyl and propofol) and the occurrence of complications during the procedure as documented by the investigator. The following patient groups without hematological malignancy were considered as immunocompromised: patients with a nonhematological malignancy who had received cytostatic chemotherapy within 30 days, solid organ transplant recipients, HIV patients with AIDS-defining illness, patients with corticosteroid-therapy with at least $10 \mathrm{mg}$ prednisone equivalent for more than 30 days or other immunosuppressive drugs (e.g., methotrexate, azathioprine, cyclosporine), pre-defined immune deficiency syndromes, and non-malignant hematological disorders with neutropenia defined as a neutrophil count below $500 / \mu \mathrm{L}$.

Complications were categorized as adverse events (AEs) and severe adverse events (SAEs) as previously described $(11,12)$. Briefly, SAEs were defined as death within $24 \mathrm{~h}$ after bronchoscopy, pneumothorax, severe bleeding (defined as necessity for intubation or placement of a bronchus blocker), need for post-interventional ventilation, epileptic seizure or any event leading to an intensive or intermediate care unit admission after the procedure. AEs were defined as any event judged as complication in the bronchoscopy report not fulfilling the definition of a severe AE, e.g., transient respiratory deterioration, short time mechanical ventilation during the procedure, hypotension, prolonged recovery after bronchoscopy as judged by the bronchoscopist, or minor bleedings.

Patient records were also screened for the results of microbiological tests in samples obtained during the procedure, e.g., bacterial or fungal cultures, aspergillus antigen detection, or molecular detection of respiratory viruses. In accordance with previous studies, the microbiological yield was defined as the percentage of procedures in which a microbiological agent could be identified (6).

\section{Statistical analysis}

Statistical analysis was performed using GraphPadPrism (GraphPad Software, La Jolla, USA). Unless otherwise stated, all data are presented as mean \pm standard deviation
(SD) after testing for normal distribution (KolmogorovSmirnov test). A two-group comparison was performed using the unpaired $t$-test for normally distributed data or the Mann-Whitney test for non-normally distributed data. The Fisher's exact test was used for categorical data. Statistical significance was defined as a $\mathrm{P}$ value $<0.05$.

\section{Results}

\section{Patient characteristics}

In the observed time period $n=268$ bronchoscopies for the evaluation of suspected pulmonary infection were performed in $201 \mathrm{HM}$ patients, whereas $\mathrm{n}=408$ bronchoscopies for the evaluation of suspected pulmonary infection were done in 371 NHM patients. Patient characteristics are summarized in Table 1. Compared to NHM patients HM patients were older (NHM: $57.5 \pm 18.0 v s$. HM: $62.9 \pm 14.4$ years; $\Delta 5.4 \pm 1.5$ years; 95\% CI, 2.5 to $8.3 ; \mathrm{P}=0.0003$ ) and had a lower prevalence of chronic lung diseases (NHM: 44.2\% vs. HM: 26.9\%; $\mathrm{P}<0.0001)$. Apart from that there were no significant differences between the groups.

At the time of bronchoscopy $39.2 \%$ of the HM group patients were neutropenic defined as a neutrophil count below $500 / \mu \mathrm{L}$, and $49.3 \%$ of the patients had a platelet count of $50 / \mathrm{nL}$ or below.

\section{Microbiological yield}

Details concerning microbiological yield are listed in Table 2, microorganisms which were frequently detected in samples obtained by bronchoscopy can be found in Table 3 . The overall microbiological yield (any bacteria, fungi or viruses) did not differ between the two patient groups (HM: $67.2 \%$ vs. NHM: 64.7\%; $\mathrm{P}=0.5622$ ). However, when cultures positive for Candida were not considered as clinically relevant the microbiological yield was significantly higher in the HM group (HM: 62.7\% vs. NHM: 53.9\%; $\mathrm{P}=0.0261)$. This difference in diagnostic yield was due to a higher detection rate of fungi (HM: $23.1 \%$ vs. NHM: $9.8 \% ; \mathrm{P}<0.0001)$ and viruses (HM: $20.2 \%$ vs. NHM: $8.8 \%$; $\mathrm{P}<0.0001)$ in the HM group whereas no differences were observed in the microbiological yield for bacteria (HM: $42.5 \%$ vs. NHM: $46.5 \% ; \mathrm{P}=0.3420$ ).

In the NHM group $\mathrm{n}=131$ procedures $(32.1 \%)$ were performed in immunocompromised patients as defined in the material and methods section. Overall diagnostic yield (any bacteria, fungi apart from Candida or viruses) in these 
Table 1 Patient characteristics

\begin{tabular}{|c|c|c|c|}
\hline Variables & $\mathrm{HM}(\mathrm{n}=201)$ & $\operatorname{NHM}(n=371)$ & $\mathrm{P}$ \\
\hline Age, years & $62.9 \pm 14.4$ & $57.5 \pm 18.0$ & $0.0003^{\#}$ \\
\hline Weight, kg & $75.3 \pm 14.5^{1}$ & $72.5 \pm 17.2^{2}$ & $0.0648^{\#}$ \\
\hline Size, $\mathrm{cm}$ & $172.4 \pm 9.9^{3}$ & $172.3 \pm 10.0^{4}$ & $0.9149^{\#}$ \\
\hline Cardio-vascular disease, n (\%) & $80(39.8 \%)$ & 148 (39.9\%) & $>0.9999^{*}$ \\
\hline Chronic pulmonary disease, $\mathrm{n}(\%)$ & $54(26.9 \%)$ & $164(44.2 \%)$ & $<0.0001^{*}$ \\
\hline \multicolumn{4}{|l|}{ Type of hematological malignancy ${ }^{7}$} \\
\hline Acute myeloid leukemia & $88(43.8 \%)$ & NA & \\
\hline Chronic lymphoblastic leukemia & $10(5.0 \%)$ & NA & \\
\hline Other & 27 (13.4\%) & NA & \\
\hline Stem cell transplantation & $49(24.4 \%)$ & NA & \\
\hline
\end{tabular}

Data are mean \pm standard deviation or number of patients (\%). ${ }^{*}$, Fisher's exact test; ${ }^{*}$, student's $t$-test; ${ }^{1}$, data were available for 191 out of 201 patients; ${ }^{2}$, data were available for 325 out of 371 patients; ${ }^{3}$, data were available for 196 out of 201 patients; ${ }^{4}$, data were available for 343 out of 371 patients; ${ }^{5}$, data were available for 131 out of 201 patients; ${ }^{6}$, data were available for 178 out of 371 patients; ${ }^{7}, 10$ patients were suffering from more than one hematological malignancy. HM, hematological malignancy; NHM, no hematological malignancy; FEV1, force expiratory volume in $1 \mathrm{~s}$.

procedures was very similar compared to the HM group (HM: $62.7 \%$ vs. immunocompromised NHM: 62.6\%; $\mathrm{P}>0.9999)$.

Interestingly, diagnostic yield for bacteria was similar in patients treated or not treated with antibiotics in both the HM (antibiotics: $45.4 \%$ vs. no antibiotics: $45.5 \%$; P>0.9999) and the NHM group (antibiotics: $42.3 \%$ vs. no antibiotics: 46.0\%; $\mathrm{P}=0.5730$ ).

In the HM group clinical management was modified (e.g., change of the antimicrobial regime or exclusion of pulmonary infection) in $36.9 \%$ of the cases due bronchoscopy results.

\section{Sedation during bronchoscopy}

Bronchoscopies in both groups were mostly performed under combined sedation. Most procedures were done using a combination of midazolam, fentanyl and propofol (HM: $63.1 \%$ vs. NHM: $61.0 \%$ ), followed by a combination of midazolam and fentanyl (HM: $17.9 \%$ vs. NHM: $17.9 \%$ ) and by a combination of midazolam and propofol (HM: 14.2\% vs. NHM: $11.8 \%)$. There were no significant differences in terms of sedation regimens between the groups $(\mathrm{P}=0.1739)$.

\section{Complications}

Details of AEs and SAEs are listed in Table 4. In general, occurrence of AEs was comparable between the two groups (HM: $14.2 \%$ vs. NHM: $15.7 \% ; \mathrm{P}=0.6580$ ) and mostly comprised transient respiratory deterioration, difficulties to adequately sedate patients and minor bleedings. The rate of minor bleedings during the procedure did not differ between hematological patients with or without thrombocytopenia ( $2.3 \%$ vs. $2.2 \%)$. In addition, SAEs rarely occurred and there were no differences in the occurrence of SAEs between the groups (HM: 2.2\% vs. NHM: $1.7 \%$; $\mathrm{P}=0.7766)$.

\section{Discussion}

This study compared diagnostic yield and safety of FOB for the diagnostic work-up of suspected pulmonary infections 
Table 2 Microbiological yield

\begin{tabular}{lccc}
\hline Microbiological yield for & $\mathrm{HM}(\mathrm{n}=268)$ & $\mathrm{NHM}(\mathrm{n}=408)$ & $\mathrm{P}$ \\
\hline Any agent & $67.2 \%$ & $64.7 \%$ & $0.5622^{*}$ \\
Any agent apart from Candida & $62.7 \%$ & $53.9 \%$ & $0.0261^{*}$ \\
Bacteria & $42.5 \%$ & $46.5 \%$ & $0.3420^{\star}$ \\
Fungi & $40.7 \%$ & $33.1 \%$ & $0.0495^{\star}$ \\
Fungi apart from Candida & $23.1 \%$ & $9.8 \%$ & $<0.0001^{*}$ \\
Viruses & $20.2 \%$ & $8.8 \%$ & $<0.0001^{*}$ \\
\hline
\end{tabular}

*, Fisher's exact test.

Table 3 Microorganisms detected in samples obtained by bronchoscopy

\begin{tabular}{|c|c|c|}
\hline Type of microorganism & $\mathrm{HM}$ & NHM \\
\hline Enterobacteriaceae $^{1}$ & $28(10.4 \%)$ & $55(13.5 \%)$ \\
\hline S. aureus & $11(4.1 \%)$ & $31(7.7 \%)$ \\
\hline$P$. aeruginosa & $5(1.9)$ & $14(3.4)$ \\
\hline Haemophilus spp. & $12(4.5 \%)$ & 35 (8.6\%) \\
\hline M. tuberculosis & $0(0 \%)$ & $23(5.6 \%)$ \\
\hline \multicolumn{3}{|l|}{ Fungi } \\
\hline Candida spp. & $63(23.5 \%)$ & $104(25.5 \%)$ \\
\hline \multicolumn{3}{|l|}{ Viruses } \\
\hline Herpes simplex & $27(10.1 \%)$ & $10(2.5 \%)$ \\
\hline Cytomegalovirus & $20(7.5 \%)$ & $15(3.7 \%)$ \\
\hline Rhinovirus & $8(3.0 \%)$ & $8(2.0 \%)$ \\
\hline Influenza virus & $6(2,2 \%)$ & $4(1.0 \%)$ \\
\hline
\end{tabular}

Data are number of positive tests (\%). ${ }^{1}$, E. coli, Proteus, Klebsiella, Enterobacter, Serratia, Citrobacter, Hafnia, Morganella.

in patients with and without underlying hematological malignancies. The overall microbiological yield was more than $60 \%$ in both groups which is in accordance with the highest results published so far $(3,5-7,13)$.

Previous studies demonstrated a considerable variability for the diagnostic yield of FOB with BAL for the identification of microbiological pathogens in hematological patients which is in the range of $26 \%$ and $65 \%(3,5-7,13)$. Factors influencing diagnostic yield and contributing to this variability include differences in empirical antimicrobial regimens, in patient populations and in the availability of methods for the detection of different pathogens, e.g., culture techniques, assays for the detection of microbiological antigens, or PCR-based techniques $(6,7)$.

Candida spp. are frequently isolated from the respiratory tract. They are mostly considered to be irrelevant as pneumonia related to Candida is an extremely rare event even in patients with hematologic malignancies (14). 
Table 4 Complications

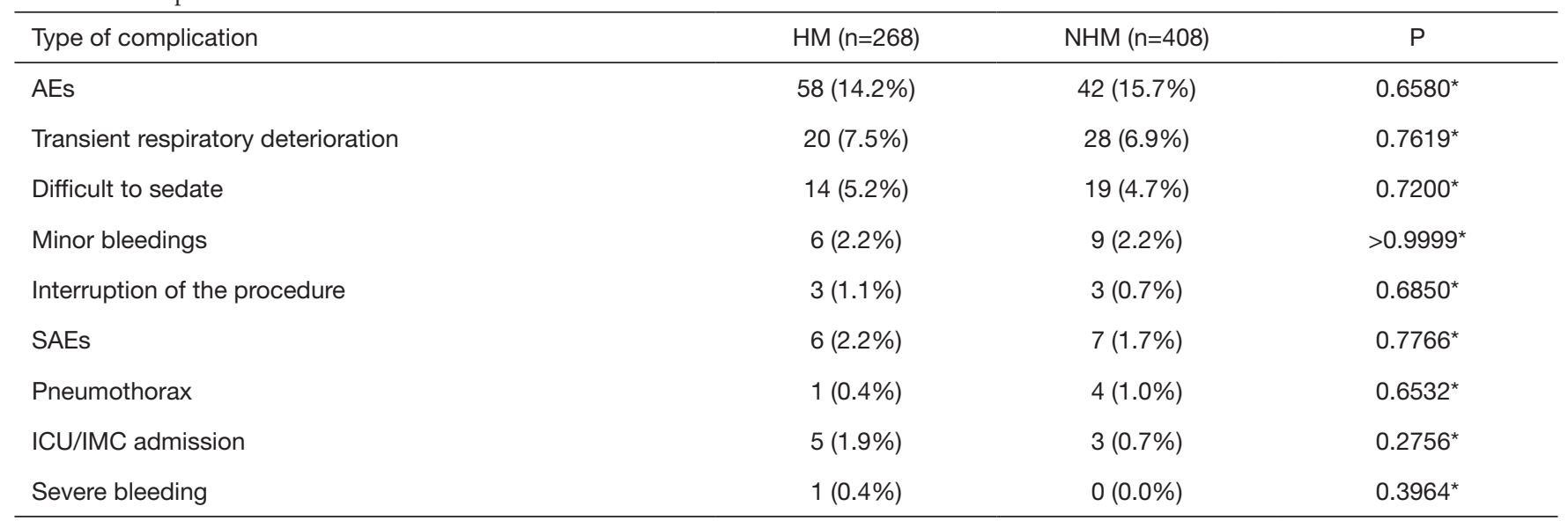

Data are number of procedures with complications (\%). *, Fisher's exact test. AE, adverse event; SAE, severe adverse event; ICU, intensive care unit; IMC, intermediate care unit.

Hence the microbiological yield was also calculated omitting cultures only positive for Candida which resulted in a higher diagnostic yield of $62.7 \%$ in the HM group compared to $53.9 \%$ in the NHM group due to a higher proportion of tests positive for viruses and fungi as expected in this patient population. Still, microorganisms apart from Candida found in respiratory samples are not necessarily of clinical relevance which is supported by the finding that bronchoscopy results lead to a change in clinical management of hematological patients in $36.9 \%$ whereas the microbiological yield was $62.7 \%$. The microbiological yield in procedures with immunocompromised patients of the NHM group was similar compared to the HM group. Consecutively, though the heterogeneity of immunocompromised non-hematological patients has to be taken into account, the presence of immunosuppression might be one possible explanation for the differences in diagnostic yield between the HM and the NHM group and emphasizes the role for FOB in diagnosing pulmonary infection especially in the context of immunosuppression.

Broad spectrum empiric antibiotic therapy should be initiated as early as possible in patients with hematological disorders when pulmonary infection is suspected especially in the context of neutropenia (15). On the other hand, this approach might decrease the microbiological yield of FOB with BAL. However, such an effect was not seen in our patient population which is in line with the results of a previous study whereas the influence of bronchoscopy results on patients' clinical management was even more pronounced than in the study done by Pagano and colleagues $(3,6)$. Consecutively, in an appropriate clinical setting bronchoscopy can provide useful information even under concurrent antimicrobial therapy.

Apart from diagnostic yield safety aspects also must be considered when performing an invasive procedure such as flexible bronchoscopy. Overall, the occurrence of complications in our study did not differ between the two groups, most complications resolved by the end of the bronchoscopy, and severe complications were rare. In the literature highly variable complication rates for flexible bronchoscopy in the management of pulmonary opacities are reported due to a lack of standardization in the documentation of complications (7). The most frequent complications in both groups were transient respiratory deterioration or problems related to sedation. Though a considerable percentage of patients in the HM group had a low platelet count, bleedings rarely occurred in both groups and most bleedings were considered as minor. Similar observations have been made previously by Nandagopal et al. who demonstrated that FOB with BAL can be safely performed in patients with thrombocytopenia (16). Taken together in accordance with most studies our results showed a favorable safety profile of flexible bronchoscopy in hematological patients $(3,5)$. Nevertheless, the potential clinical benefit of the results obtained by bronchoscopy should always be balanced against the risks of the procedure especially in patients with respiratory failure (4).

This analysis has several limitations which need to be addressed. Firstly, the data were not obtained prospectively. Therefore, bronchoscopies and the diagnostic work- 
up of microbiological samples were not performed according to a standardized protocol in the same way in all patients. For the same reason there was no standardized assessment of a patient's cardiopulmonary status prior bronchoscopy. Secondly, there were some differences in patient characteristics, e.g., patients in the NHM group were younger and had a higher prevalence of chronic lung diseases. Finally, we had to rely on patient records to determine the occurrence of AEs and SAEs. All these factors could lead to bias when comparing complication rates between the groups. These limitations could only be overcome by a randomized clinical trial comparing clinical management and outcome in patients undergoing or not undergoing flexible bronchoscopy for the diagnosis of pulmonary infections, though the conception of such a study would be very difficult.

Despite these shortcomings, this study, performed in a large patient cohort for the first time showed that diagnostic yield and the occurrence of complications were comparable when performing flexible bronchoscopy for the investigation of pulmonary infection both in patients with and without underlying hematological malignancy.

\section{Acknowledgments}

Funding: None.

\section{Footnote}

Data Sharing Statement: Available at http://dx.doi. org/10.21037/jtd-20-835

Peer Review File: Available at http://dx.doi.org/10.21037/jtd20-835

Conflicts of Interest: All authors have completed the ICMJE uniform disclosure form (available at http://dx.doi. org/10.21037/jtd-20-835). The authors have no conflicts of interest to declare.

Ethical Statement: The authors are accountable for all aspects of the work in ensuring that questions related to the accuracy or integrity of any part of the work are appropriately investigated and resolved. Data analysis was done with regard to the Declaration of Helsinki (as revised in 2013). The Institutional Ethical Review Board for Human Studies at RWTH ("Rheinisch-Westfälische Technische Hochschule") University was involved and confirmed that a formal approval was not required as this retrospective analysis required neither an intervention nor irregularity of privacy or anonymity (EK 099/17). Individual patient consent was waived by the Review Board due to the observational nature of the study.

Open Access Statement: This is an Open Access article distributed in accordance with the Creative Commons Attribution-NonCommercial-NoDerivs 4.0 International License (CC BY-NC-ND 4.0), which permits the noncommercial replication and distribution of the article with the strict proviso that no changes or edits are made and the original work is properly cited (including links to both the formal publication through the relevant DOI and the license). See: https://creativecommons.org/licenses/by-nc-nd/4.0/.

\section{References}

1. Maschmeyer G, Donnelly JP. How to manage lung infiltrates in adults suffering from haematological malignancies outside allogeneic haematopoietic stem cell transplantation. Br J Haematol 2016;173:179-89.

2. Chaoui $\mathrm{D}$, Legrand $\mathrm{O}$, Roche $\mathrm{N}$, et al. Incidence and prognostic value of respiratory events in acute leukemia. Leukemia 2004;18:670-5.

3. Pagano L, Pagliari G, Basso A, et al. The role of bronchoalveolar lavage in the microbiological diagnosis of pneumonia in patients with haematological malignancies. Ann Med 1997;29:535-40.

4. Bauer PR, Chevret S, Yadav H, et al. Diagnosis and outcome of acute respiratory failure in immunocompromised patients after bronchoscopy. Eur Respir J 2019;54:1802442.

5. Makita K, Mikami Y, Matsuzaki H, et al. Utility of bronchoscopy in the definitive diagnosis of patients with haematological malignancies presenting with radiological abnormalities. Clin Respir J 2018;12:1381-8.

6. Sakata KK, Klassen CL, Bollin KB, et al. Microbiologic yield of bronchoalveolar lavage specimens from stem cell transplant recipients. Transpl Infect Dis 2017;19:e12684.

7. Choo R, Anantham D. Role of bronchoalveolar lavage in the management of immunocompromised patients with pulmonary infiltrates. Ann Transl Med 2019;7:49.

8. Ruhnke M, Behre G, Buchheidt D, et al. Diagnosis of invasive fungal diseases in haematology and oncology: 2018 update of the recommendations of the infectious diseases working party of the German society for hematology and medical oncology (AGIHO). Mycoses 2018;61:796-813. 
9. Ruhnke M, Böhme A, Buchheidt D, et al. Diagnosis of invasive fungal infections in hematology and oncology-guidelines from the Infectious Diseases Working Party in Haematology and Oncology of the German Society for Haematology and Oncology (AGIHO). Ann Oncol 2012;23:823-33

10. Maschmeyer G, Carratalà J, Buchheidt D, et al. Diagnosis and antimicrobial therapy of lung infiltrates in febrile neutropenic patients (allogeneic SCT excluded): updated guidelines of the Infectious Diseases Working Party (AGIHO) of the German Society of Hematology and Medical Oncology (DGHO). Ann Oncol 2015;26:21-33.

11. Müller T, Thümmel K, Cornelissen CG, et al. Analogosedation during flexible bronchoscopy using a combination of midazolam, propofol and fentanyl - A retrospective analysis. PLoS One 2017;12:e0175394.

12. Cornelissen CG, Dapper J, Dreher M, et al. Endobronchial ultrasound-guided transbronchial needle aspiration under general anesthesia versus bronchoscopistdirected deep sedation: A retrospective analysis. Endosc

Cite this article as: Panse J, von Schwanewede K, Jost E, Dreher M, Müller T. Pulmonary infections in patients with and without hematological malignancies: diagnostic yield and safety of flexible bronchoscopy - a retrospective analysis. J Thorac Dis 2020;12(9):4860-4867. doi: 10.21037/jtd-20-835
Ultrasound 2019;8:204-8.

13. Kim SW, Rhee CK, Kang HS, et al. Diagnostic value of bronchoscopy in patients with hematologic malignancy and pulmonary infiltrates. Ann Hematol 2015;94:153-9.

14. Meersseman W, Lagrou K, Spriet I, et al. Significance of the isolation of Candida species from airway samples in critically ill patients: a prospective, autopsy study. Intensive Care Med 2009;35:1526-31.

15. Link H, Böhme A, Cornely OA, et al. Antimicrobial therapy of unexplained fever in neutropenic patients-guidelines of the Infectious Diseases Working Party (AGIHO) of the German Society of Hematology and Oncology (DGHO), Study Group Interventional Therapy of Unexplained Fever, Arbeitsgemeinschaft Supportivmassnahmen in der Onkologie (ASO) of the Deutsche Krebsgesellschaft (DKG-German Cancer Society). Ann Hematol 2003;82 Suppl 2:S105-17.

16. Nandagopal L, Veeraputhiran M, Jain T, et al. Bronchoscopy can be done safely in patients with thrombocytopenia. Transfusion 2016;56:344-8. 